\title{
Diffractive optical elements for the production of synthetic spectra
}

\author{
M.B. Sinclair, M.A. Butler, A.J. Ricco, S.H. Kravitz and W.J. Zubrzyckí \\ Sandia National Laboratories, Albuquerque, NM 87185-1405

\begin{abstract}
We demonstrate that computer-generated diffractive optical elements can be used to synthesize the infrared spectra of real compounds. In particular, we describe a modified phase-retrieval algorithm that we have used to design diffractive elements of this type and we present experimental results for a diffractive optic which is capable of synthesizing the infrared spectrum of HF between $3600 \mathrm{~cm}^{-1}$ and $4300 \mathrm{~cm}^{-1}$. The reflection-mode diffractive optic consists of 4096 lines, each $4.5 \mu \mathrm{m}$ wide, at 16 discrete depths relative to the substrate (from 0 to $1.2 \mu \mathrm{m}$ ), and was fabricated on a silicon wafer using anisotropic reactive ion-beam etching in a four-mask-level process. We propose the use of such elements to replace reference cells in a new type of correlation spectroscopy that we call "holographic correlation spectroscopy." Storage of a large number of diffractive elements, each producing a synthetic spectrum corresponding to a different target compound, in compact disk-like format, will allow a spectrometer of this type to rapidly determine the composition of unknown samples. Further, this approach can be used to perform correlation-based measurements of hazardous or transient species, for which conventional correlation spectroscopy is impractical.
\end{abstract}

Keywords: synthetic spectra, correlation spectroscopy, diffractive optics, infrared spectroscopy

\section{INTRODUCTION}

Continued refinement of semiconductor microlithographic techniques has stimulated increasing interest in diffractive optics, since it is now possible to accurately fabricate large-area surface relief patterns containing subwavelength features. Advancement of diffractive optic technology is dependent on progress in two primary areas: development of an understanding of the capabilities of diffractive optics, so that new applications can be identified; and improvement of the procedures used to design these devices, to enable high performance devices. ${ }^{1,2}$ Although several mulispectral applications have been investigated previously, the bulk of the development effort has thus far been directed toward the development of diffractive optics for monochromatic applications. In this paper, we describe a new application for diffractive optics: the production of synthetic infrared spectra. In addition, we propose the use of these elements to produce reference spectra for a new type of correlation spectroscopy that we call "holographic correlation spectroscopy." "This paper is organized as follows: first, a simple discussion of correlation spectroscopy is presented to enumerate the advantages of using diffractive optics in this technique; second, the computational procedure used for the design of diffractive optics that are capable of synthesizing infrared spectra are outlined; finally, the performance of a diffractive optic which was designed and fabricated to synthesize the infrared spectrum of HF between $3600 \mathrm{~cm}^{-1}$ and $4300 \mathrm{~cm}^{-1}$ is presented.

\section{CORRELATION SPECTROSCOPY}

Correlation spectroscopy is an attractive technique for sensing and analysis applications because it combines the attributes of mechanical and optical simplicity, high selectivity, and good sensitivity. ${ }^{4-6}$ In correlation spectroscopy, the degree of correlation between the transmission of an unknown sample and a reference cell containing a known compound is determined over a fixed spectral range. ${ }^{4}$ For materials such as gases that are
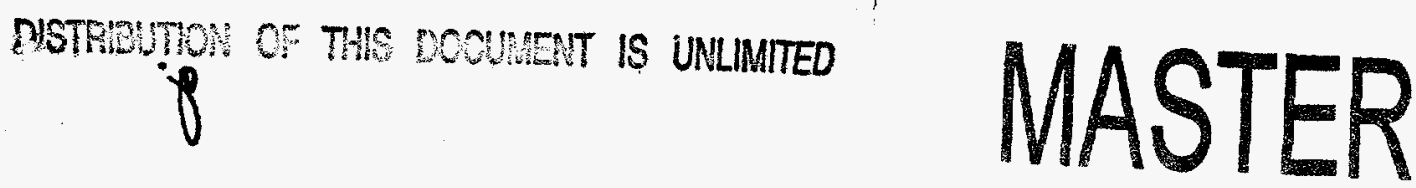


\section{DISCLAMMER}

Portions of this document may be illegible in electronic image products. Images are produced from the best available original document. 
characterized by narrow absorption lines, the cross-correlation between the transmission spectra of different compounds can usually be neglected, ${ }^{4}$ and a significant correlation will be observed only when the sample and reference cells contain the same compound. Excellent selectivity is thereby obtained. Correlation spectroscopy also achieves good sensitivity for materials with narrow absorption bands, since the transmission auto-correlation functions of these materials are sharp and small modulations of the transmission of the reference cell can impose a large modulation on a beam that transits both cells.

In a typical correlation spectrometer, broadband light first passes through the unknown sample, and then through a reference cell containing a target compound (see Fig. la). ${ }^{4-6}$ To measure the degree of correlation between the two spectra, the transmission spectrum of the reference cell is modulated by a suitable means, ${ }^{4-6}$ and the output of the detector at the modulation frequency is recorded. In the case where the modulation of the reference cell transmission is equivalent to a wavelength shift by an amount $\delta \lambda$, the fractional modulation of the transmitted optical power $\left(\delta \mathrm{P} / \mathrm{P}_{0}\right)$ recorded at the detector will be given by

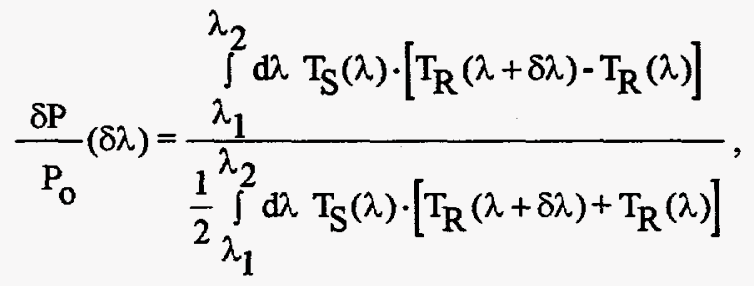

where $T_{S}(\lambda)$ and $T_{R}(\lambda)$ are the transmission spectra of the sample and reference cells, respectively. For simplicity, the spectrum of the incident radiation is assumed to be constant in the range from $\lambda_{1}$ to $\lambda_{2}$ and zero elsewhere.

a)



b)

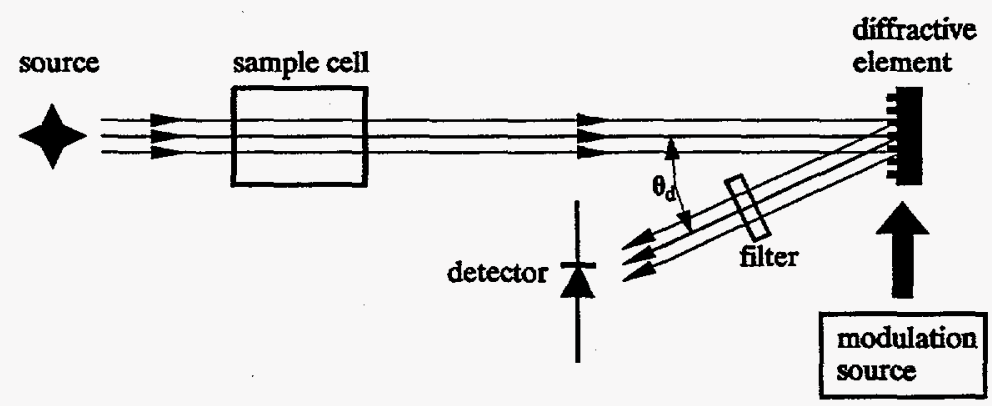

Figure 1. a)A schematic representation of a traditional correlation spectrometer.

b)A schematic representation of a holographic correlation spectrometer. 
For high selectivity and good sensitivity, it is essential that the spectral range used in the determination of the cross-correlation is large enough to encompass several absorption bands of the target compound. When, both the sample and reference cells contain the same compounds, the cross-correlation of their transmission functions will be strongly peaked at $\delta \lambda=0$, and a small wavelength modulation of the transmission of the reference cell can lead to a relatively large fractional modulation of the transmitted power. Conversely, when the target compound is not present in the sample cell, the cross-correlation is not peaked at $\delta \lambda=0$, and the fractional modulation will be small.

The transmission spectra for many compounds of interest are characterized by narrow, isolated, absorption bands and are thus near unity for most wavelengths. For these materials, a large background of unmodulated radiation will reach the detector since the overall transmission will only be modulated in spectral regions where the reference absorbs. Consequently, the denominator of Eq. 1 will be large, and the magnitude of the fractional modulation of the transmitted light will be limited. To overcome this difficulty, the total spectrum of light that is allowed to reach the detector is usually limited by means of a band-pass filter to one or more regions near important absorption bands.

Although this discussion assumes a wavelength modulation of the reference transmission, other modulation schemes (such as pressure modulation and Stark modulation) that result in line width modulation of the reference transmission are more commonly used. A third type of modulation is achieved by phase modulating the optical radiation as it propagates from the sample to the reference. ${ }^{6}$ An important characteristic of any modulation method is maintaining a constant spectrally integrated transmission of the reference cell to minimize any background modulation of the transmitted light.

A schematic diagram of the holographic correlation spectrometer is shown in Figure $1 \mathrm{~b}$. The principal difference between this spectrometer and the traditional correlation spectrometer of Fig. 1a is the replacement of the reference cell by a diffractive element. The diffractive element may operate in either a reflective (shown) or transmissive mode. In this configuration, the total power measured at a diffraction angle $\theta_{\mathrm{d}}$ is determined by the cross-correlation between the transmission of the sample cell, and the diffraction efficiency of the diffractive element (at $\theta_{d}$ ). If the diffraction efficiency spectrum closely corresponds to the absorption spectrum of a compound of interest, then the effect of the diffractive element is analogous to that arising from a reference cell containing a physical sample.

The use of diffractive elements to produce reference spectra has several significant advantages over the use of reference cells containing real materials. ${ }^{7}$

1. The diffractive elements can be extremely compact, allowing many to be stored on a common substrate. Alternatively, a single, programmable diffraction grating may be used to recreate the spectra of a large number of materials. Thus, a correlation spectrometer relying on synthetic spectra can easily be configured to detect many different compounds.

2. The diffractive elements may be designed to only reproduce a subset of the target spectrum. This is desirable in situations where interference from overlapping absorption of other non-target compounds is expected. By reproducing only those portions of the target spectrum that are free from chemical interferences, increased selectivity will be obtained.

3. The diffracted light spectrum may be modulated in ways not possible with real reference materials. In the case of simple wavelength modulation, the magnitude of the modulation may be made to be significantly larger than can be achieved with a real reference material. More complex forms of modulation may also be used. In this way both the sensitivity and selectivity of the spectrometer may be optimized for a given application. Once again, for all modulation schemes, it is essential that the spectrally integrated diffraction efficiency remains constant to avoid spurious intensity modulation of the transmitted optical energy.

4. Diffractive elements may be designed to simulate dangerous species such as chemical warfare agents or corrosive compounds, or transient chemical species whose lifetimes are too short to allow their use as reference materials. 
5. Since there is spatial information in the direction perpendicular to the diffraction plane, it should be possible to develop an imaging correlation spectrometer by modifying a simple holographic correlation spectrometer to include a detector array and "push broom" collection optics.

A final advantage of the use of diffractive elements in correlation spectroscopy is obtained when the reference spectrum synthesized is the complement $\left(1-T_{R}\right)$ of the transmission $\left(T_{R}\right)$ of the real target compound. In this case the fractional change in the detected power is given by

$$
\frac{\delta P}{P_{0}}(\delta \lambda)=\frac{-\int_{\lambda_{1}}^{\lambda_{2}} d \lambda T_{S}(\lambda) \cdot\left[\mathrm{T}_{R}(\lambda+\delta \lambda)-\mathrm{T}_{R}(\lambda)\right]}{\frac{1}{2} \int_{\lambda_{1}}^{\lambda_{2}} \mathrm{~d} \lambda \mathrm{T}_{S}(\lambda) \cdot\left[\mathrm{T}_{\mathrm{R}}(\lambda+\delta \lambda)+\mathrm{T}_{\mathrm{R}}(\lambda)-2\right]} .
$$

Note that, except for a change in sign, the numerator of Eq. 2 is identical to that of Eq. 1. However, the denominator of Eq. 2 will be significantly smaller than that of Eq. 1 when the transmission spectra of the sample and reference compounds consist of narrow, isolated lines. Thus, the fractional modulation of the detected power (and, hence, the signal-to-noise ratio) is larger when the reference spectrum is the complement of the transmission spectrum of the target compound.

\section{DESIGN PROCEDURES}

The production of a synthetic spectrum requires the design of a diffractive element, whose diffraction efficiency spectrum at a predetermined diffraction angle is identical to the infrared spectrum of interest. For a phase-shifting element, this requires the determination of a phase profile, $\phi(x)$, which, when imposed on the incident broadband infrared beam, will result in the desired efficiency spectrum. The procedure used to design these optics was described in detail in Ref. 7, and will only be outlined briefly here. First, the infrared spectrum of the compound of interest is converted to a " $u$-space" amplitude profile, where $u$ is the Fourier-space variable conjugate to the linear coordinate along the diffractive element $(\mathrm{x})$. Assuming normal incidence on the diffractive element, $u$ is given by:

$$
\mathbf{u}=\frac{\sin (\theta)}{\lambda}
$$

where $\theta$ is the diffraction angle, and $\lambda$ is the wavelength of the incident light. In contrast to monochromatic applications, which exploit the angular dependence of $u$, the present application exploits its spectral dependence. Working in the Fraunhofer approximation ${ }^{8}$ and assuming spatially uniform incident radiation, a modified Gerchberg-Saxton algorithm ${ }^{9,10}$ is then used to obtain a phase profile $\phi(x)$ which produces a close approximation to the desired $\mathrm{u}$-space profile. To accommodate a four-mask-level fabrication procedure, the traditional GerchbergSaxton algorithm is modified to produce a phase profile discretized to 16 phase levels. The phase profile determined in this manner is then translated into a surface relief profile, $d(x)$, using $d(x)=\phi(x) \cdot \lambda_{1} / 4 \pi$, where $\lambda_{1}$ is a specific wavelength within the spectral range of interest (usually the wavelength which corresponds to the largest feature in the spectrum). The surface relief profile obtained in this fashion represents the first approximation of the solution to the design problem. For any wavelength $(\lambda)$, the phase profile imposed by the surface relief profile determined above is given by:

$$
\phi_{\lambda}(x)=\left[\frac{4 \pi}{\lambda}\right] \cdot d(x)
$$

where the subscript indicates that the phase profile depends parametrically on the wavelength. Note that the imposed phase profile will exactly match the phase profile determined by the Gerchberg-Saxton algorithm only at $\lambda_{1}$. 
The spectrum of diffracted radiation is obtained from the surface relief profile by calculating the diffracted intensity for each wavelength within the spectral range of interest. For each wavelength, the phase profile imposed by the diffractive element is calculated according to Eq. 4, and a forward Fourier transform is performed to generate the $u$-space profile for that wavelength. Since both $\lambda$ and $\theta$ are known, the u-space coordinate required for the output spectrum is determined from Eq. 3, and the amplitude corresponding to this $u$-value can be extracted from the u-space profile. This procedure is then repeated for each wavelength in the desired spectral range to assemble the entire spectrum. The surface relief profile is further optimized using a simulated quenching procedure, ${ }^{11}$ in which the full spectrum is calculated for each step in the quenching algorithm.

\section{DEVICE FABRICATION AND EVALUATION}

The target compound for this investigation, hydrogen fluoride, was chosen for several reasons. ${ }^{12}$ First, the IR spectrum of $\mathrm{HF}$ in the region from $3600 \mathrm{~cm}^{-1}$ to $4300 \mathrm{~cm}^{-1}$ consists of a series of sharp rotational lines superimposed on a broad vibrational envelope. The multi-line nature of this spectrum makes $\mathrm{HF}$ an ideal candidate for detection using correlation spectroscopy. ${ }^{4}$ Hydrogen fluoride is also an example of a compound for which construction of a stable reference is difficult and hazardous. A solid-state diffractive optic that synthesizes the IR spectra of HF would circumvent this problem, providing the basis for a correlation spectrometer for the detection of HF. Using the procedure described above and in Ref. 7, a reflection-mode diffractive optic was designed to synthesize the HF spectrum at a diffraction angle of $15^{\circ}$. It consists of 4096 lines, each of width 4.5 $\mu \mathrm{m}$, for an overall length of $18.4 \mathrm{~mm}$. The depths of the lines relative to the substrate ranges from 0 to $1.2 \mu \mathrm{m}$ in 16 discrete steps. The choice of 4096 lines is dictated by the desire to achieve high spectral resolution (which requires a large element with many lines), without introducing significant computational or fabrication complications.

The surface relief profile was fabricated on a silicon wafer using a four-mask-level process (since the etching process described below is additive, $n$ mask levels can be used to produce $2^{n}$ discrete etch depths) ${ }^{12}$. The four masks were produced on quartz substrates using direct-write electron-beam lithography. For each mask level, the silicon wafer was treated with a vapor of hexamethyldisilazane (HMDS), and a 1.4- $\mu \mathrm{m}$-thick layer of AZ5214 photoresist was applied by spin coating. Alignment of the $2^{\text {nd }}, 3^{\text {rd }}$, and $4^{\text {th }}$ mask levels to the previous levels was accomplished using a Suss MJB3 contact printer. The mask corresponding to the shallowest etch depth was used first, with subsequent masks used in order of increasing etch depths. Alignment features were included in the mask design to aid in this process. Mask-to-mask alignment tolerances are estimated to be $\pm 0.5 \mu \mathrm{m}$. Subsequent to development of the photoresist images, the silicon was etched in a load-locked reactive-ion-beam etcher described elsewhere. ${ }^{13}$ This machine produces extremely anisotropic silicon etching, with $\pm 5 \%$ depth uniformity. After the $4^{\text {th }}$ level was etched, a reflective layer consisting of $100 \AA$ of titanium and $500 \AA$ of gold is deposited onto the diffractive element. Figure 2 shows a scanning electron micrograph of a portion of one of the gratings produced in this manner.



Figure 2. A scanning electron micrograph of an end view of a portion of the HF element. 
A diagram of the experimental apparatus used to evaluate the diffractive elements is shown in Figure $3 .^{12}$ The output of a tungsten-halogen lamp is directed through an order-sorting filter and chopper, and focused onto the entrance slit of a $0.25 \mathrm{~m}$ monochromator. The output of the monochromator is collimated with a $200 \mathrm{~mm}$ focal length spherical mirror, directed onto a flat steering mirror, and then strikes the diffractive element at normal incidence. The infrared radiation diffracted at $15^{\circ}$ to the element normal is collected by a $200 \mathrm{~mm}$ focal length mirror and focused through an adjustable slit onto a photoconductive $\mathrm{PbS}$ detector. The detector output was recorded using a lock-in amplifier. A personal computer was used to scan the monochromator and record the output of the lock-in amplifier. The intensity spectrum recorded in this manner is normalized by replacing the diffractive element with a flat mirror which is adjusted to reflect the infrared radiation in the direction of the focusing mirror. In this manner, diffraction efficiency spectra are obtained.

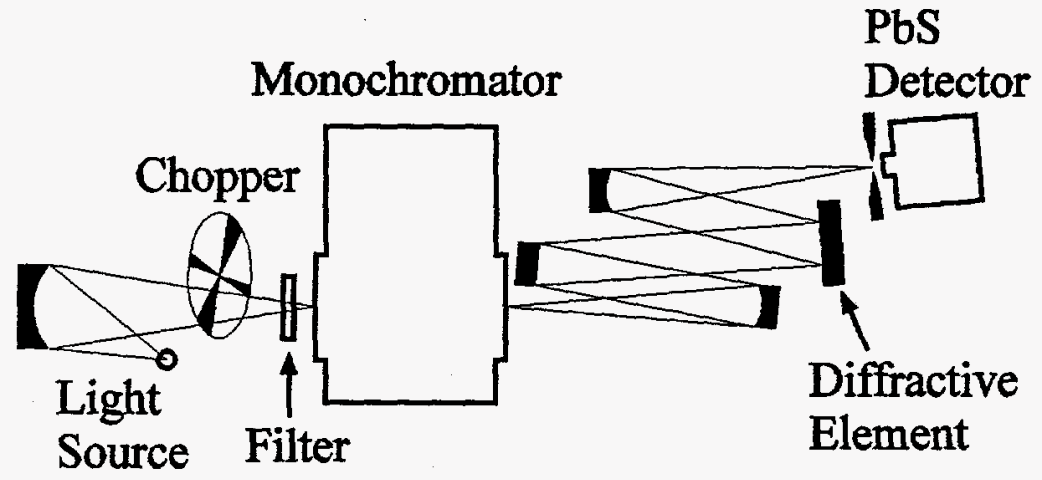

Figure 3. A diagram of the experimental apparatus used to record the diffraction efficiency spectrum of the diffractive optical element.

The infrared spectrum of HF in the region between $3600 \mathrm{~cm}^{-1}$ and $4250 \mathrm{~cm}^{-1}$ is shown in Figure $4 \mathrm{a}^{14}$ Apart from an increase in the widths of the rotational lines, the theoretical diffraction efficiency spectrum predicted by the algorithm (Fig. 4b) closely resembles the real HF spectrum. The increased line width is due to the resolution limitations imposed by the use of an $18.4 \mathrm{~mm}$ diffractive element. With a larger element, narrower absorption lines could be synthesized. Figure $4 \mathrm{c}$ shows the synthetic spectrum measured from the HF diffractive element. ${ }^{12}$ All the major rotational lines, seen in the real HF spectrum, appear in the synthetic spectrum at the correct spectral positions and with approximately correct relative intensities. Thus, this optical element successfully synthesizes the general features of the HF spectrum. The spectral positions of the diffraction efficiency peaks observed for the $\mathrm{HF}$ diffractive element match those of the rotational lines of $\mathrm{HF}$ only at a $15^{\circ}$ diffraction angle. Small variations in the angle at which the diffracted radiation is observed result in shape-preserving spectral shifts of $\sim 300 \mathrm{~cm}^{-1} / \mathrm{deg}$. This behavior is precisely what is expected from consideration of the diffraction process (see Eq. 1), and offers an easy means of generating the spectral modulation required for correlation spectroscopy.

Although the synthetic spectrum shown in Fig. 4c faithfully reproduces many of the features of the real HF spectrum (Fig. 4a), it is obvious that the synthetic spectrum exhibits deviations from the real spectrum. The primary deviation is the increased line width of the synthetic spectrum. This is principally due to the resolution limitations of the simple experimental apparatus used to record the synthetic spectrum. Narrower lines have been obtained by increasing the resolution of the monochromator, at a cost of decreased signal-to-noise levels. The line widths of the spectrum shown here represent a compromise between resolution and signal-to-noise considerations. The development of a characterization system capable of higher resolution and better signal-to-noise levels is 


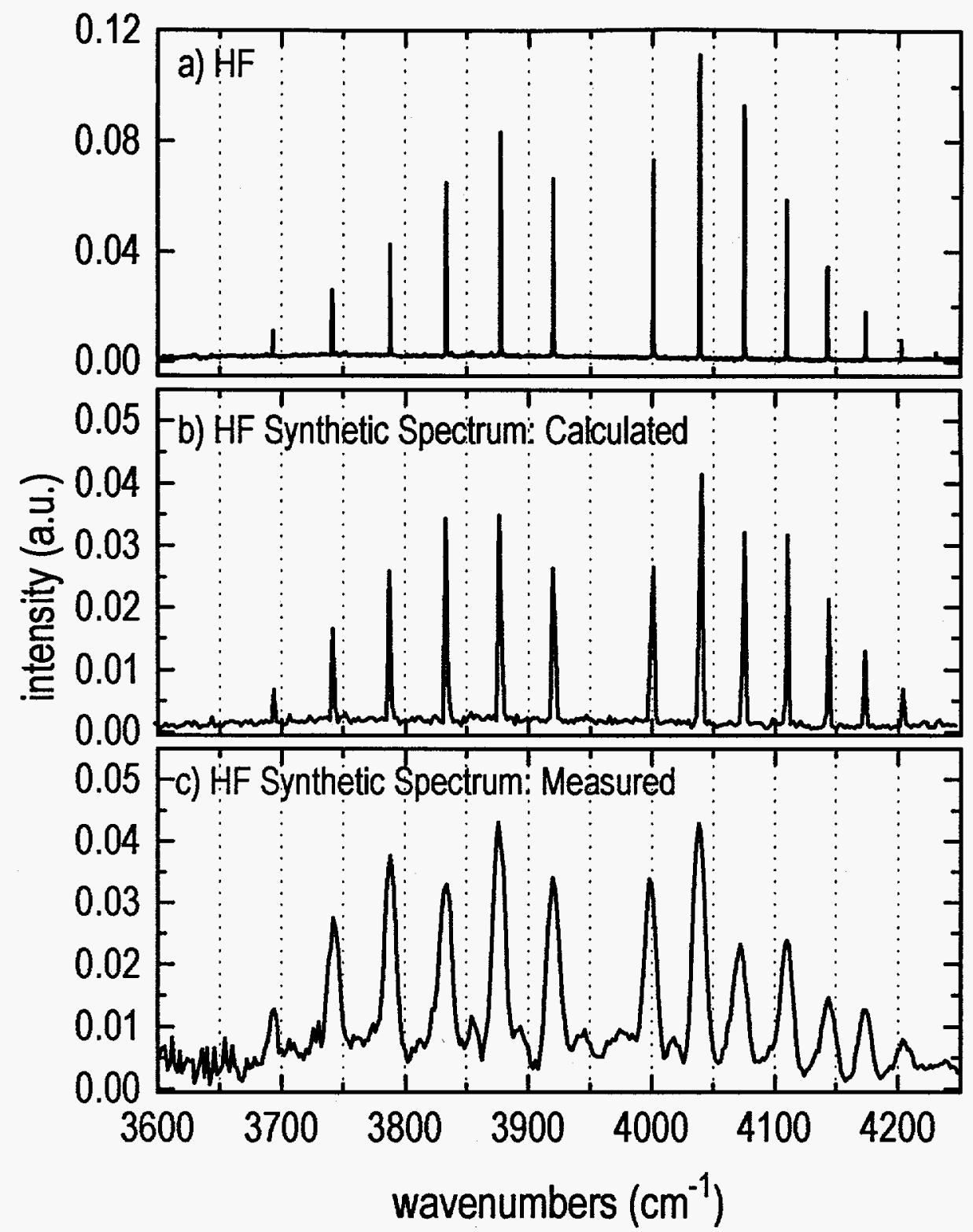

Figure 4. (a) The infrared spectrum of HF between $3600 \mathrm{~cm}^{-1}$ and $4250 \mathrm{~cm}^{-1}$.

(b) The theoretical synthetic spectrum predicted by the design algorithm.

(c) The Infrared spectrum measured for the diffractive element shown in Fig. 1.

currently under way. It is important to note that slightly broadened lines are not necessarily detrimental to correlation spectroscopy applications: provided the extent of spectral modulation exceeds the width of the synthetic spectral lines, the magnitude of the correlation signal will be very similar to that obtained using a perfect spectral match.

The other significant discrepancies between the synthetic spectrum and the real spectrum are the appearance of small features (peaks, shoulders, etc.) between the rotational lines, as well as small deviations of the relative intensities from the real spectrum. Although the origin of these features has not yet been pinpointed, several likely candidates exist and are being investigated. First, the accuracy of the scalar diffraction method used 
to design the diffractive element is questionable, since the minimum feature size is only twice as large as the operating wavelength. It is expected that higher accuracy will be obtained by incorporating rigorous diffraction models $^{1,2}$ in the design of the element. Second, fabrication errors (i.e. mask alignment errors and etch depth errors) result in deviations from the as-designed profile, and are expected to result in discrepancies between the synthetic and real spectra. Finally, any spatial non-uniformity of the infrared radiation incident on the diffractive element may adversely affect spectral fidelity.

\section{CONCLUSIONS}

We have developed a computational procedure that allows the design of diffractive elements that can be used to synthesize the infrared spectra of real materials. In addition, we have designed and fabricated a diffractive optic that successfully synthesizes the HF spectrum between $3600 \mathrm{~cm}^{-1}$ and $4300 \mathrm{~cm}^{-1}$. To our knowledge, this represents the first demonstration of using diffractive optics to synthesize infrared spectra. Finally, we have described a holographic correlation spectrometer in which the reference spectra are generated using diffractive elements rather than reference cells. Many of the limitations imposed by the use of real reference materials, such as those associated with toxic, corrosive, or transient species, are avoided using synthetic spectra. Storage of a large number of diffractive elements in compact disk-like format, or use of a programmable diffractive element, will allow a spectrometer of this type to detect many compounds without the need for reconfiguration. At present, work is underway to refine the design algorithms and improve our fabrication procedures in an effort to improve the accuracy of the synthetic spectra, and to demonstrate the use of these novel diffractive elements in a correlation spectroscopy-based chemical sensor system.

\section{ACKNOWLEDGMENTS}

This work was supported by the United States Department of Energy under Contract DE-AC04-94AL85000. Sandia is a multiprogram laboratory operated by Sandia Corporation, a Lockheed Martin Company, for the United States Department of Energy.

\section{REFERENCES}

1. J. Opt. Soc. Am., A12, \#5,1995, (special issue on diffractive optics).

2. Appl. Opt., 34, \#14, 1995, (special issue on diffractive optics).

3. The adjective "holographic" is used not because holography is used to produce the diffractive elements, but because like white-light holograms, the diffractive elements are designed to simultaneously diffract several wavelengths of light at a common diffraction angle.

4. R. Goody, "Cross-Correlating Spectrometer," J. Opt. Soc. Am., 58, 900-908, 1968.

5. H.O. Edwards and J.P. Daikin, "Gas sensors using correlation spectroscopy compatible with fibre-optic operation," Sensors and Actuators, B11, 9-19, 1993.

6. D.M. Rider, J.T. Schofield, J.S. Margolis, and D.J. McCleese, "Electrooptic phase modulation gas correlation spectroscopy: a laboratory demonstration," Appl. Opt 25, 2860-2862, 1986.

7. M.B. Sinclair, M.A. Butler, A.J. Ricco, and S.D. Senturia, "Synthetic spectra: a new tool for correlation spectroscopy," Appl. Opt., in press.

8. J.W. Goodman, Introduction to Fourier Optics, Chap. 4, p. 57-74, McGraw-Hill, 1968.

9. R.W. Gerchberg and W.O. Saxton, "A practical algorithm for the determination of phase from image and diffraction plane pictures," Optik 35, 237-246, 1972. 
10. J.R. Fienup, "Phase retrieval algorithms: a comparison," Applied Optics 21, 2758-2769, 1982.

11. D.W. Prather, M.S. Mirotznik, and J.N. Mait, "Design of subwavelength diffractive optical elements using a hybrid finite element-boundary element method," Proc. SPIE, 2689, 14-23, 1996.

12. M.B. Sinclair, M.A. Butler, S.H. Kravitz, W. Zubrzycki, and A.J. Ricco, "Synthetic infrared spectra," Opt. Lett., (submitted).

13. G. Allen Vawter, Joel R. Wendt, "Chlorine reactive ion-beam etching of InSb and $\operatorname{InAs_{0.15}} \mathrm{Sb}_{0.85} / \mathrm{InSb}$ strained-layer superlattices," Appl. Phys. Lett., 58, 289-291, 1991.

14. The infrared spectrum of HF was obtained from: Infrared Analysis, Inc., 1334 North Knollwood Circle, Anaheim, CA, 92801. 